\title{
MedienPädagogik
}

Zeitschrift für Theorie und Praxis der Medienbildung

www.medienpaed.com

ISSN 1424-3636

Jahrbuch Medienpädagogik 17:

Lernen mit und über Medien in einer digitalen Welt

Herausgegeben von Klaus Rummler, Ilka Koppel, Sandra Aßmann,

Patrick Bettinger und Karsten D. Wolf

\section{Resonanzstrategien im Umgang mit Beschleunigung und Digitalisierung}

Jana Wienberg und Klaus Buddeberg

\section{Zusammenfassung}

Die Fragestellung des Beitrags betrifft Lernen und Kompetenzentwicklung Erwachsener im Kontext von Beschleunigung und Digitalisierung. Der Beitrag diskutiert dieses Phänomen auf theoretischer und empirischer Ebene. Vor dem Hintergrund zeitgenössischer Diagnosen zeichnet sich eine zunehmende Individualisierung (Beck 1986), Modernisierung (Field, zit. n. Hösel u.a. 2017), Wettbewerbs-/ Optimierungslogik (Kessl 2017; Rosa 2016a; 2016b), Beschleunigung (Rosa 2005) und Digitalisierung (Aufenanger 2018; Käpplinger 2016; Burchert und Grobe 2017; Schmid, Goetz und Behrens 2017) ab, welche u.U. zu erhöhten Bestrebungen nach (Selbst-)Optimierung führen. So lässt sich der Bedeutungszuwachs als Folge einer zunehmenden Komplexität des heutigen Lebens deuten (Ribolits 2007), gekennzeichnet durch eine Zunahme von Informationen und «informational overload», verstärkte Unkalkulierbarkeit auf den Arbeitsmärkten sowie eine bereits seit den 2000er Jahren «steigende Unsicherheit in den (berufs-)biografischen Perspektiven» (Arbeitsstab Forum Bildung 2001). Gleichzeitig - das zeigen Ergebnisse einer bevölkerungsrepräsentativen Erhebung in Deutschland - sehen sich verschiedene Bevölkerungsgruppen (z.B. formal niedrig Gebildete, gering Literalisierte, Arbeitslose, Ältere) digitalen Herausforderungen ausgesetzt, für die sie nach eigener Auffassung nur über geringe Kompetenzen verfügen. Das gilt sowohl für funktional-pragmatische Kompetenzen (Klieme und Hartig 2008) als auch für kritischhinterfragende Kompetenzen (Negt 1993; Zeuner 2009, 268-69; Riekmann und Grotlüschen 2011, 69). Exemplarische Ergebnisse werden im Beitrag präsentiert. 


\title{
Relational resonance strategies related to acceleration and digitization
}

\begin{abstract}
The question of the contribution concerns learning and competence development of adults in the context of acceleration and digitization. The paper discusses this phenomenon on a theoretical and empirical level. Against the background of contemporary diagnoses, increasing individualization (Beck 1986), modernization (Field, cited by Hösel et al. 2017), competition/optimization logic (Kessl 2017; Rosa 2016a; 2016b), acceleration (Rosa 2005) and digitization (Aufenanger 2018; Käpplinger 2016; Burchert and Grobe 2017; Schmid, Goetz and Behrens 2017) are emerging, which may lead to increased (self-)optimisation efforts. For example, the increase in importance can be interpreted as a consequence of the increasing complexity of life today (Ribolits 2007), characterized by an increase in information and "informational overload», increased incalculability on the labor markets and, since the 2000s, «increasing uncertainty in (occupational) biographical perspectives» (Arbeitsstab Forum Bildung 2001). At the same time - this is shown by the results of a representative population survey in Germany various population groups (e.g. formally low-educated, low literate, unemployed, elderly) face digital challenges for which they themselves consider that they have only little competence. This applies both to functional-pragmatic competences (Klieme and Hartig 2008) and to critical-questioning competences (Negt 1993; Zeuner 2009, 268-69; Riekmann and Grotlüschen 2011, 69). Exemplary results are presented in the article.
\end{abstract}

\section{Einleitung}

Unser Beitrag thematisiert die komplexe Verflechtung zwischen Digitalisierung, Beschleunigung bis hin zur Selbstoptimierung: Die Theorie der Beschleunigung (King und Gerisch 2009; Rosa 2014; Rosa 2009; Korunka und Kubicek 2013; Paech 2012) geht davon aus, dass unsere heutige Gesellschaft einem allumfassenden Beschleunigungsphänomen unterliegt, das unweigerlich dazu führt, dass «alles immer schneller und immer mehr wird», wodurch sich die Individuen zunehmend in Zeitnot fühlen. Es 
scheint, dass sich der «hochtourige Lerner zum turbobedingten Prototyp des effektiven Selbstlerners» (Meyer-Drawe 2008, 125) entwickelt hat und damit einhergehend Schnelligkeit zu einem unbefragten Konkurrenzprinzip im Spannungsfeld des Lehr-Lern-Geschehens geworden ist. Hierbei wird häufig Zeitknappheit trotz Zeitgewinn durch technische Beschleunigung wahrgenommen.

Auf pragmatischer Ebene kann die Aneignung technokratischer Methoden zur Priorisierung, zum Zeitsparen, zur Effizienzsteigerung und Optimierung von Tätigkeiten und Handlungsverläufen in der Zeit gesehen werden (Schmidt-Lauff 2012, 52-53). Hierbei zeichnet sich ein Aufkommen neuer Zeitkonkurrenzen zwischen Erwerbstätigkeit, Lernen und privater Zeitverwendung sowie eine Auflösung der Dichotomie und klaren Trennung zwischen Arbeiten und Lernen ab (Schmidt-Lauff 2004, 125); hervorgehend aus (beschleunigtem) digitalem Wandel im Kontext von Arbeit und Beschäftigung. Parallel zu den beschriebenen Beschleunigungstendenzen entwickelt sich eine zunehmende Vernetzung, die den Umgang mit Zeit und Raum verändert. Diese Entwicklungen in Richtung einer wachsenden Flexibilisierung und Technologisierung/Digitalisierung zeichnen sich lebensbereichsübergreifend ab.

Auf theoretischer Ebene wurde eine Weiterentwicklung des Resonanzachsen-Modells (nach Rosa 2016b) vorgenommen. Auf empirischer Basis wurde unter resonanztheoretischer Perspektive die Frage aufgeworfen, welche Resonanz- und Entfremdungspotenziale sich identifizieren lassen (Wienberg 2018; Wienberg 2019a). Hierzu wurden leitfadengestützte Interviews mit Teilnehmenden aus Weiterbildungsveranstaltungen aus dem Bereich der betrieblichen Weiterbildung und der konfessionellen Erwachsenenbildung durchgeführt. Im Zuge dessen wurden temporale Resonanzstrategien im Umgang mit Beschleunigung/Digitalisierung (Vorlaufen Mitlaufen - Innehalten - Gegenlaufen) aus den Daten generiert (Wienberg 2018; 2019a). Das entwickelte heuristische Modell der Relationalen Resonanzstrategien (RRS) dient der Beschreibung von erlebter Resonanz und Entfremdung im Lehr-Lern-Geschehen unter Bedingungen einer beschleunigten, digitalisierten spätmodernen Gesellschaft, die Subjekte, so wie anhand der empirischen Befunde zu digitalen Kompetenzen gezeigt werden kann, an die Grenzen ihrer selbstberichteten Kompetenzen führen können. 


\section{Ausgangspunkte}

\subsection{Wie gut sind Erwachsene auf digitale Herausforderungen vorbereitet?}

Aufgrund von Beschleunigungstendenzen erscheinen Innovationszyklen sich zu verkürzen. Die damit einhergehende Digitalisierung/Technologisierung drückt sich für Teile der Gesellschaft eher als ein informational overload`denn als Verheissung aus. Dieses Phänomen kann zu zunehmenden «Entscheidungslasten» und «Unsicherheiten» in modernen Gesellschaften führen und als ein Grund für die steigende Komplexität des heutigen Lebens gesehen werden (Ribolits 2007).

Aus einer bildungswissenschaftlichen Perspektive stellt sich die Frage, wie Individuen und Gesellschaften auf diese Veränderungen vorbereitet sind. Eine Reihe von Kompetenzstudien fokussiert dabei die digitalen Kompetenzen von Jugendlichen und von Erwachsenen. Im Herbst 2019 wurden in Washington D.C. die Ergebnisse der auf Jugendliche bezogene ICILS-Studie 2018 (International Computer and Information Literacy Study) der Öffentlichkeit vorgestellt und via Internet weltweit übertragen. ${ }^{1}$ Die Resultate liegen als international vergleichender Bericht (Fraillon et al. 2019) und als Länderbericht für Deutschland vor (Eickelmann et al. 2019). Obwohl sich die Studie auf Schülerinnen und Schüler bezieht, sollen drei inhaltliche Befunde Erwähnung finden, die sich auch auf die Fragen dieses Beitrags beziehen, der sich mit dem Umgang von Erwachsenen mit digitaler Technologie befasst. Zunächst betonte Studiendirektor Julian Fraillon bei der Vorstellung der Ergebnisse, dass die internationalen Unterschiede deutlich geringer sind als die Leistungsunterschiede innerhalb der teilnehmenden Länder (Fraillon et al. 2019, 74; Eickelmann et al. 2019, 123). Dadurch gewinnt die ausführliche Analyse von nationalen Strukturdaten an Bedeutung gegenüber dem internationalen Vergleich. Als weiteres Resultat stellte Fraillon die Annahme in Zweifel, junge Menschen würden allein dadurch, dass sie im stetigen Kontakt mit digitaler Technologie und mit digitalen Geräten aufwachsen, profunde digitale Kompetenzen entwickeln (Fraillon et al. 2019, 242), dadurch also, dass sie - als komplette Generation

1 https://www.iea.nl/news-events/events/release-icils-2018-results. 
- digital natives sind (zur kritischen Diskussion des Begriffs vgl. Schulmeister 2012; Kammer 2018; Pietraß 2013). Und schliesslich deuten die grossen Leistungsunterschiede innerhalb der Länder auf eine breite Streuung digitaler Kompetenzen hin (Fraillon et al. 2019, 244). Bezogen auf die untersuchte Gruppe von Schülerinnen und Schüler in Deutschland bedeutet dies: «Weniger als ein Viertel (22.0\%) der Achtklässlerinnen und Achtklässler in Deutschland erreicht Kompetenzen, die der Kompetenzstufe IV entsprechen» (Eickelmann et al. 2019, 131). Auch die nachrückende Generation, wie sie in ICILS einem Benchmark unterzogen wird, dürfte zu grösseren Teilen also auch im Erwachsenenalter eher eingeschränkte Kenntnisse im Umgang mit digitaler Technologie haben. Für Erwachsene zwischen 16 und 65 Jahren hat die erste Runde der PIAAC-Studie (Programme for the International Assessment of Adult Competencies) für die Mehrzahl der teilnehmenden Länder - darunter Deutschland - nur kleine Anteile von Erwachsenen mit ausdrücklich hoher digitaler Kompetenz ermittelt (Wicht et al. 2018; Wolf und Koppel 2017). PIAAC hat neben der Lesekompetenz und der alltagsmathematischen Kompetenz auch das Problemlösen in technologiereichen Umgebungen untersucht (zum theoretischen Konstrukt vgl. Zabal et al. 2013, 61-63; Lennon und Tamassia 2016, 9-11).

Digitale Kompetenzen wurden auch in der LEO-Studie der Universität Hamburg untersucht. Die Studie basiert auf der Befragung eines bevölkerungsrepräsentativen Samples von 7.192 Deutsch sprechenden Erwachsenen im Alter von 18 bis 64 Jahren (Grotlüschen und Buddeberg 2020). Sie erfasste im Rahmen der Nationalen Dekade für Alphabetisierung und Grundbildung (BMBF und Kultusministerkonferenz 2016) die Lese- und Schreibkompetenz von Erwachsenen in Deutschland. Darüber hinaus betrachtete die Studie spezifische Alltagspraktiken und Grundkompetenzen von Erwachsenen in verschiedenen Domänen, darunter auch im Kontext Digitalisierung.

Die folgende ausschnittartige Darstellung von Ergebnissen verfolgt das Ziel, Aspekte von Digitalisierung zu illustrieren, die von Teilen der erwachsenen Bevölkerung als voraussetzungsreich und möglicherweise schwer zu bewältigen wahrgenommen werden. Es existiert eine Reihe von Alltagspraktiken, die direkt oder indirekt mit dem Einsatz digitaler Technologie zusammenhängen, an denen aber Teil der erwachsenen 
Bevölkerung nur bedingt teilhaben (ausführliche Diskussion in Buddeberg und Grotlüschen 2020). Das betrifft bezogen auf digitale Alltagspraktiken beispielsweise die selbstständige Nutzung des Internets. Laut LEO-Studie nehmen 26 Prozent der Erwachsenen (Anteilsangaben jeweils gerundet) zumindest gelegentlich Unterstützung bei der Internetnutzung in Anspruch. Darüber hinaus nutzen 24 Prozent das Internet nicht, um nach gesundheitsbezogenen Informationen zu suchen, sich mit Hilfe von Onlinetutorials oder Lehrvideos zu informieren (25 Prozent) oder Bankgeschäfte zu erledigen (31 Prozent). Am Beispiel des Onlinebankings werden gruppenspezifische Unterschiede sichtbar. Unter den 55- bis 64-Jährigen liegt der Anteil derer, die kein Onlinebanking betreiben, bei 45 Prozent, unter Personen mit niedriger formaler Bildung bei 47 Prozent und unter Erwachsenen, die Schwierigkeiten beim Schreiben oder Erfassen zusammenhängender Texte haben (gering literalisierte Erwachsene), bei 55 Prozent. Obwohl also digitale Technologie vielfach als ubiquitär beschrieben wird, nehmen unterschiedliche Bevölkerungsgruppen diese Technologien in Form alltäglicher digitaler Praktiken durchaus unterschiedlich in Anspruch. Offen bleibt in dieser Betrachtungsweise, ob seltene Praktik mit geringer Kompetenz einhergeht. Zwar ist ein allgemeiner Zusammenhang zwischen der Lesekompetenz und der alltäglichen Lese- und Schreibpraxis auf Basis der PIAAC-Studie belegt (Reder 2017), ob aber die Nicht-Nutzung von Onlinebanking nicht auch ein Ausweis ausgeprägter Reflexion und einer bewussten kritischen Entscheidung gegen diese Technologie ist, lässt sich ebenfalls vermuten.

Unterschiede werden auch deutlich hinsichtlich der selbstzugeschriebenen Kompetenz, bestimmte Onlinedienste zu nutzen bzw. Aspekte von Digitalisierung kritisch zu beurteilen. So trauen sich Erwachsene auf einer funktional-pragmatischen Ebene zwar weitreichende Kompetenzen zu, weniger ausgeprägt ist das Zutrauen in die eigene Kompetenz aber bezogen auf kritisch-hinterfragende Kompetenzen. Funktional-pragmatische Kompetenzen lassen sich in Anlehnung an Klieme und Leutner als «kontextspezifische kognitive Leistungsdispositionen» verstehen, «die sich funktional auf Situationen und Anforderungen in bestimmten Domänen beziehen» (Klieme und Leutner 2006, 879). In Anlehnung an Negt richten sich darüber hinausreichende gesellschaftliche Kompetenzen darauf, 
Angelegenheiten und Zusammenhänge kritisch beleuchten und hinterfragen zu können (Negt 1990). Auf funktional-pragmatischer Ebene geben jeweils rund zehn Prozent der Erwachsenen an, dass sie bei der Nutzung von Online-Stellenbörsen bzw. von Online-Wohnungsbörsen grössere Schwierigkeiten zu erwarten hätten. Der Zusammenhang zwischen diesen selbstberichteten Kompetenzen und dem Alter ist moderat, ein stärkerer Zusammenhang besteht hingegen zur formalen Bildung (Tabelle 1).

\begin{tabular}{|l|l|l|l|}
\hline $\begin{array}{l}\text { Anteil von Erwachsenen, die es } \\
\text { sich nicht oder nur mit grösseren } \\
\text { Schwierigkeiten zutrauen... }\end{array}$ & $\begin{array}{l}\text { alle Erwach- } \\
\text { sene }\end{array}$ & $\begin{array}{l}\text { Erwachsene } \\
\text { ohne oder } \\
\text { mit niedri- } \\
\text { gem Schul- } \\
\text { abschluss }\end{array}$ & $\begin{array}{l}\text { ältere Er- } \\
\text { wachsene } \\
\text { (55-64 } \\
\text { Jahre) }\end{array}$ \\
\hline Online-Stellenbörsen zu nutzen & $10,2 \%$ & $24,4 \%$ & $15,1 \%$ \\
\hline Online-Partnerportale zu nutzen & $13,5 \%$ & $27,6 \%$ & $18,9 \%$ \\
\hline Online-Wohnungsportale zu nutzen & $10,4 \%$ & $22,6 \%$ & $15,7 \%$ \\
\hline
\end{tabular}

Tab. 1.: Funktional-pragmatische digitale Kompetenzen von Erwachsenen. Quelle: Universität Hamburg, LEO 2018 - Leben mit geringer Literalität, Basis 6894 Deutsch sprechende Erwachsene (18-64 Jahre).

Auf der Ebene kritisch-hinterfragender Kompetenzen hingegen steigt der Anteil der Erwachsenen, die sich hier Schwierigkeiten ausgesetzt sehen (Tabelle 2). Das gilt für die selbsteingeschätzte Kompetenz, sich mit der Glaubwürdigkeit von Informationen im Internet kritisch auseinanderzusetzen oder bei Texten im Internet zwischen Information und Werbung zu unterscheiden. Rund 22 Prozent der Erwachsenen halten es für schwierig oder eher schwierig, die Glaubwürdigkeit von Informationen im Internet zu beurteilen, rund 22 Prozent erachten es als (eher) schwierig, zu unterscheiden, ob es sich bei Texten im Internet um Informationen oder um Werbung handelt. Korrespondierend mit diesen Ergebnissen ergab eine Untersuchung unter Jugendlichen in Schweden eine geringe Kompetenz des Fact-Checkings, aufschlussreicherweise besonders bei den Jugendlichen, die sich hierbei selbst besonders hohe Kompetenzen zuschrieben (Nygren und Guath 2019). 
Besonders deutliche Schwierigkeiten geben Erwachsene dabei an, die Motive zu erkennen und zu beurteilen, die der Sammlung personenbezogener Daten im Internet zugrunde liegen. Rund 43 Prozent der Erwachsenen halten dies für (eher) schwierig. Auch hinsichtlich dieser Aspekte ist der Zusammenhang mit dem Alter moderat, ein deutlicher Zusammenhang besteht aber auch hier zum Niveau der formalen Bildung.

\begin{tabular}{|l|l|l|l|}
\hline $\begin{array}{l}\text { Anteil von Erwachsenen, die es } \\
\text { für sich als (eher) schwierig be- } \\
\text { trachten... }\end{array}$ & $\begin{array}{l}\text { alle Erwach- } \\
\text { sene }\end{array}$ & $\begin{array}{l}\text { Erwachsene } \\
\text { ohne oder } \\
\text { mit niedri- } \\
\text { gem Schul- } \\
\text { abschluss }\end{array}$ & $\begin{array}{l}\text { ältere Er- } \\
\text { wachsene } \\
\text { (55-64 Jah- } \\
\text { re) }\end{array}$ \\
\hline $\begin{array}{l}\text { die Glaubwürdigkeit von Informa- } \\
\text { tionen im Internet zu beurteilen }\end{array}$ & $21,5 \%$ & $35,8 \%$ & $25,2 \%$ \\
\hline $\begin{array}{l}\text { bei Texten im Internet Information } \\
\text { von Werbung zu unterscheiden }\end{array}$ & $22,2 \%$ & $40,9 \%$ & $25,7 \%$ \\
\hline $\begin{array}{l}\text { die Motive von IT-Unternehmen } \\
\text { für die Sammlung von Daten der } \\
\text { Nutzenden zu beurteilen }\end{array}$ & $42,5 \%$ & $56,3 \%$ & $46,0 \%$ \\
\hline
\end{tabular}

Tab. 2.: Kritisch-hinterfragende digitale Kompetenzen von Erwachsenen. Quelle: Universität Hamburg, LEO 2018 - Leben mit geringer Literalität, Basis 6894 Deutsch sprechende Erwachsene (18-64 Jahre).

Diese selbstberichteten Kompetenzen verweisen jedoch auf ein allenthalben bestehendes Problembewusstsein. Insofern kann die Schwierigkeit, den Wahrheitsgehalt von Informationen zu beurteilen, auch als grundsätzlicher Beleg kritischer Medienkompetenz gelesen werden.

Digitalisierung stellt also, so lassen sich die bisherigen Ausführungen zusammenfassen, einen Prozess dar, in dem sich Gesellschaften grundsätzlich befinden, an dem aber nicht alle Teile der jeweiligen Bevölkerung in gleichem Masse teilhaben (digitale Praktiken), und bezogen auf den sich verschiedene Bevölkerungsgruppen in der Selbstauskunft nur geringe Kompetenzen zuschreiben (digitale Grundkompetenzen), bzw. für den ein grundsätzliches Problembewusstsein unterstellt werden kann. Unterschiedliche Bevölkerungsgruppen differieren also hinsichtlich ihrer Voraussetzungen, mit einer als Herausforderung wahrnehmbaren 
Digitalisierung umzugehen. Welche Strategien können Erwachsene als Reaktion darauf entwickeln, um mit diesen Herausforderungen durch Digitalisierung (und Beschleunigung) umzugehen?

\subsection{Entfremdung und Resonanz im Umgang mit Beschleuni- gung und Digitalisierung}

Der Beschleunigungstheorie zufolge sind moderne Gesellschaften nach Rosa (2014) einem allumfassenden Beschleunigungsphänomen unterlegen - insbesondere vor dem Hintergrund einer wachsenden Technologisierung und Digitalisierung durch u.a. die rasante Steigerung von Mobilität und Kommunikationsmöglichkeiten und einer damit einhergehenden grossen zeitlichen und räumlichen Flexibilisierung. Hierbei können mögliche Interdependenzen im Spannungsfeld zwischen Kultur und Technik u.a. im Hinblick auf das Wissensmanagement eine zentrale Rolle einnehmen. Mit dieser lebensbereichsübergreifenden Beschleunigung und einer zunehmenden Technologisierung kann auch auf individueller Ebene ein umfassender sozialer, kultureller und ökonomischer Wandel einhergehen (Blossfeld et al. 2017). Beispielsweise können sich Veränderungen von sozialen Praktiken, Kommunikationsstrukturen und Lebensformen abzeichnen (Rosa 2016b, 42f.). Zudem können sich durch eine vermeintliche Beschleunigung des Lebenstempos Steigerungs- und Wettbewerbslogik verschärfen und zugleich die Optionenvielfalt erhöhen.

Parallel zu Beschleunigungstendenzen entwickelt sich eine zunehmende Vernetzung, die den Umgang mit Zeit und Raum verändert. Dies zeigt sich in der Geschwindigkeit von Neuentwicklungen, dem Obsolet-Werden scheinbar überholter Technologien, Datenübertragung und -verarbeitung in grossen Mengen sowie immer flexibler werdenden Arbeitsorten. Dadurch veraltet das erlangte Wissen immer schneller, wodurch die Erstausbildung häufig nicht mehr ausreicht, um am Arbeitsmarkt erfolgreich bestehen zu können: Das Lernen im Erwachsenenalter müsste sich folglich zu einem Normalstand entwickeln, einhergehend mit einem Ausbau von Weiterbildungsstrukturen, höheren Qualifizierungen sowie Umschulungen (Institut für Arbeitsmarkt- und Berufsforschung (IAB 2019, 37). Diese Entwicklungen sind jedoch nicht ausschliesslich technikgetrieben, sondern 
können mit dem Konzept des soziotechnischen Systems als Ausdruck der Wechselwirkungen und Abhängigkeiten von Technik, Mensch und Organisation verstanden und beschrieben werden (Ahrens und Gessler 2018; Hirsch-Kreinsen 2016) und sind damit angebunden an gesellschaftliche Entwicklungen. Die möglicherweise hierbei auftretenden Interdependenzen können im Spannungsfeld zwischen Kultur und Technik vor dem Hintergrund einer zunehmenden Beschleunigung und Digitalisierung eine zentrale Rolle einnehmen. Die Kehrseite der zunehmenden Digitalisierung könnte eine Entfremdung durch Technik und durch einen Überfluss an Kommunikationsangeboten darstellen. Angesichts einer steigenden Technologisierung, Automatisierung und damit häufig einhergehenden Rationalisierungstendenz lässt sich eine ambivalente Entwicklung zwischen Dequalifizierung einerseits und Tätigkeitsanreicherung andererseits identifizieren (Benjamin 1991, 610; Rosa 2012). Mit der Entwicklung neuer Technologien gehen demzufolge veränderte (berufliche) Tätigkeitsprofile einher (IAB 2019, 7). Somit erhöht sich in der Weiterbildung der Bedarf nach übergreifenden Kompetenzen, die sich aus digitalen, sozialen sowie fächerübergreifenden Kompetenzen zusammensetzen (IAB 2019, 11). Eine Rollen- bzw. Aufgabenverschiebung ist ebenfalls bei den Lehrenden in der Erwachsenenbildung/Weiterbildung sichtbar, da sich ihr Tätigkeitsprofil weg von der Weiterbildungsorganisation und hin zur Prozessberatung/moderation entwickelt (Haberzeth und Sgier 2019).

Rosas Aussage folgend, «[...] wenn Beschleunigung das Problem ist, dann ist Resonanz vielleicht die Lösung»(Rosa 2016b, 13), soll jenes Beschleunigungsphänomen vor dem Hintergrund erlebter Resonanz und Entfremdung im Folgenden betrachtet und eingangs die zentralen Begriffe skizziert werden:

Auf theoretischer Ebene kann Entfremdung als

«[...] eine spezifische Form der Weltbeziehung, in der Subjekt und Welt einander indifferent oder feindlich (repulsiv) und mithin innerlich unverbunden gegenüberstehen [...]. Entfremdung definiert damit einen Zustand, in dem die 〈Weltanverwandlung〉 misslingt, so dass die Welt stets kalt, starr, abweisend und nicht-responsiv erscheint $[. ..] \gg($ Rosa 2016a, 316). 
Rosa fasst damit jegliche Formen gestörter Weltbeziehungen der Subjekte als Entfremdung zusammen. Diese sind gekennzeichnet durch das Fehlen konstitutiver, responsiver Beziehungen sowie fehlende Selbstwirksamkeits- und Kontrollüberzeugungen und die Abwesenheit handlungsorientierender, positiver Bindungen zur Welt. Er umschreibt somit einen

«Zustand [...], in welchem Subjekte Ziele verfolgen oder Praktiken ausüben, die ihnen einerseits nicht von anderen Akteuren oder äußeren Faktoren aufgezwungen wurden [...] welche sie aber andererseits nicht, wirklich' wollen oder unterstützen» (Rosa 2011, 234).

Hervorzuheben ist dabei, dass Rosa in Abgrenzung zu z.B. (vor-)modernen Entfremdungstheorien, in denen das konsumtive Auskosten des (kapitalistischen) Ertrags kein Element der Lebensführung darstellt (Weber 2016), den Kapitalismus als zentrales Mittel sieht, um die Eckpfeiler moderner Vorstellungen eines gelingenden Lebens, nämlich Autonomie und Authentizität, verwirklichen zu können. In diesem Deutungsansatz definieren insbesondere die Beschleunigung und das Wachstum als kulturprägende und strukturbildende Kräfte eine kapitalistische Gesellschaftsordnung. Rosas Theorie lässt sich darüber hinaus in Abgrenzung zu (vor-) modernen Entfremdungstheorien - in denen u.a. Kritik an Arbeits- und Produktionsweisen als Vergegenständlichungsprozess (Marx 1996), an einer Reduzierung der Zuschreibung von (Objekt-)Werten auf eine reine Quantitätsdifferenz (Simmel 1919), an Rationalisierungsprozessen (Weber 2016), an der entindividualisierenden und vermassenden Wirkung der Populärkultur bzw. der technischen Rationalität (Horkheimer und Adorno 1969; Marcuse 1998) geübt wurde - als eine Übertragung des (vor-)modernen Entfremdungsbegriffs auf postmoderne Gesellschaften in Zeiten von Dienstleistungen und Digitalisierung sowie Big Data ansehen. Postmoderne Entfremdungstheorien, wie auch Rosas Ansatz, thematisieren zum einen stärker die zunehmende Rationalisierung und Uniformität der (Massen-)Gesellschaft i.S. einer totalen Homogenisierung (Ritzer 2017); zum anderen kritisieren sie im Hinblick auf Entfremdung den rationalisierten Gebrauch von Zeit, welcher durch Diskontinuität (Ungewissheit, Flexibilität) geprägt ist (Sennett 1998; Jaeggi 2005: 63ff.). 
Der hier verwendete Entfremdungsbegriff kann in einer kritischen Betrachtung von Bedingungen unseres Zusammenlebens als ein Deutungsmuster begriffen werden, welches dazu beiträgt, Phänomene in der Welt zu erschliessen, zu interpretieren sowie zu beurteilen.

Das Pendant zur Entfremdung bildet der Resonanzbegriff: Resonanz stellt demnach

«[...] eine durch Affizierung und Emotion, intrinsisches Interesse und Selbstwirksamkeitserwartung gebildete Form der Weltbeziehung [dar], in der sich Subjekt und Welt gegenseitig berühren und zugleich transformieren [...]. Resonanz ist [somit] keine Echo-, sondern eine Antwortbeziehung [...]. Resonanz ist demzufolge kein emotionaler Zustand, sondern ein Beziehungsmodus. Dieser ist gegenüber dem emotionalen Inhalt neutral» (Rosa 2016a, 298).

So lassen sich Entfremdung und Resonanz nicht als Gegensätze deuten, sondern vielmehr als komplementäre Beziehungsmodi, welche in Bildungs- und Anwandlungsprozessen in ein dialektisches Verhältnis treten (Beljan 2017, 397; Renn 2009, 593f.). Vor diesem Hintergrund kann Bildung als ein dynamischer Prozess begriffen werden, welcher sowohl resonante als auch entfremdende Momente implizieren muss (Rosa 2016a, 316). Resonanz kann als ein antwortender und (gegebenenfalls) transformierender Prozess begriffen werden, wobei er eine Unterscheidung zwischen «stummen〉 (das heisst gleichgültigen oder latent feindlichen) und 〈resonanten〉 Weltverhältnissen vornimmt (Baer 2005). Erstgenannte〈stummen〉Verhältnisse sind häufig rein mechanische, instrumentelle und kausale Weltbeziehungen, in denen kein Interesse in der Auseinandersetzung entwickelt bzw. das Subjekt nicht begeistert und berührt wird. Stumme Weltverhältnisse können - im Gegensatz zu resonanten Beziehungen, die als energiegenerierend angesehen werden - als «energiezehrend» bezeichnet werden, da der zu erbringende Aufwand als belastend empfunden wird und dadurch das Individuum andernorts (in Freizeit, Familie, Sport und/ oder Natur) diesen «Energieverlust» wieder kompensieren muss (Beljan 2017, 398). Im Hinblick auf die Differenzierung zwischen zuvor genannten 〈stummen〉 und 〈resonanten〉 Weltverhältnissen lässt sich auf die Vertreterin der zeitgenössischen Kritischen Theorie, Rahel Jaeggi, Bezug nehmen 
(Jaeggi in Rosa 2016a). Sie begreift Lebensformen als ein Ensemble sozialer Praktiken, welche direktional auf die Problemlösung gerichtet sind (Jaeggi 2014).

Es ist fraglich, welche Rolle die Technik bei Wechselwirkungen mit dem Körper und den Sinnen einnimmt und welche Ausprägungen räumliche und zeitliche Aspekte der Digitalisierung und der Beschleunigung auf das Lehr-Lern-Geschehen haben. Einige Autorinnen und Autoren diagnostizieren im beruflichen Kontext eine Tendenz, Arbeitnehmende implizit mit einem „körperlosen Selbst” zu denken oder zumindest der Psyche gegenüber dem Körper viel mehr Gewicht einzuräumen (Lengersdorf 2017, 19f.; Gärtner und Ortmann 2017, 176f.).

\subsection{Empirischer Zugang}

Es wird die Frage aufgeworfen, welche Resonanz- und Entfremdungspotenziale sich aus Sicht von Weiterbildungsteilnehmenden empirisch identifizieren lassen.

Die zentrale Fragestellung der Untersuchung lautet: Wie stellen sich zum einen erlebte Momente von Resonanz und Entfremdung sowie deren dialektisches Wechselspiel im Lehr-Lern-Geschehen aus Teilnehmendensicht in der Erwachsenenbildung/Weiterbildung dar, und wie gestaltet sich der individuelle Umgang damit?

Ein weiteres Untersuchungsziel stellt die Betrachtung von Phänomenen und Aspekten des Lernens unter Bedingungen von Beschleunigung und Digitalisierung dar. Resonanz bildet einen Rahmen, der helfen kann, Umgangsweisen und Subjekt-Umwelt-Beziehungen nachvollziehbar gestalten und systematisieren zu können. Ziel ist es jedoch, weniger den Wandel vom Analogen zum Digitalen zu beschreiben, als vielmehr die unterschiedlichen Qualitäten in Bezug auf Resonanz und Entfremdung zu rekonstruieren, die wiederum Aufschlüsse geben über mögliche Veränderungen des Lehr-Lern-Verhältnisses im Hinblick auf ein Digital-AnalogKontinuum aus Sicht der Teilnehmenden in der Erwachsenenbildung/Weiterbildung. 
Hierzu wurden leitfadengestützte Interviews $(\mathrm{N}=20)$ mit Teilnehmenden eines betrieblichen Weiterbildungsanbieters (Global Player) und eines konfessionellen Erwachsenenbildungsanbieters durchgeführt, die Daten nach Grounded Theory (Strauss und Corbin 1996) ausgewertet sowie Kodier-Familien (Strategien) nach Glaser (1978) gebildet. Als Erkenntnis konnte die Herausbildung Relationaler Resonanzstrategien (RRS) u.a. im Umgang mit Beschleunigung und Digitalisierung gewonnen werden. Hierbei konnten Momente der Reflexion bzw. Bewusstwerdung von Resonanzerfahrungen sowie von Widersprüchen und Entfremdung sichtbar gemacht sowie kollektive und individuelle RRS im Lehr-Lern-Geschehen aus Teilnehmendensicht identifiziert werden.

Hierbei handelt es sich um eine retroperspektive Beschreibung der Befragten. Die leitfadengestützten Interviews sind, neben einem einleitenden und einem abschliessenden Frageteil, in drei übergeordnete Themenblöcke unterteilt:

1. Soziale Beschleunigung und Digitalisierung,

2. Resonanz und

3. Entfremdung.

Die Kategorien (2) Resonanz und (3) Entfremdung implizieren jeweils die Aspekte einer

- stofflichen Dimension,

- performanten, interaktionalen und didaktischen Dimension,

- leiblichen/körperlichen Dimension

im Lehr-Lern-Geschehen aus Teilnehmendensicht.

Jene Fragen werden vor dem Hintergrund einer sozialen Beschleunigung und zunehmenden Digitalisierung sowie einer damit verbundenen Veränderung der Wahrnehmung und dem Erleben zeitlicher und räumlicher Dimensionen («Reichweitenvergrösserung», «Gegenwartsschrumpfung») gestellt. Ebenso werden die Themenbereiche Resonanz und Entfremdung in ihren unterschiedlichen Bezügen - im Umgang mit Stofflichkeit, Leiblichkeit sowie in der Performanz und Interaktion im konkreten Weiterbildungsgeschehen - thematisiert. In diesem Zusammenhang werden Fragen nach der Art und Weise formuliert, in denen Menschen in Beziehung 
zur Welt treten, sie erfahren und wahrnehmen, in ihr handeln und sich in ihr orientieren. Durch einen weiteren Fragetypus, den im Leitfaden eingebauten Konfrontationsfragen bzw. die Veranlassung zum gedanklichen Perspektivwechsel, können die bis dahin erfolgten Schilderungen der Befragten noch einmal im Licht der Kontrastierung erlebter Momente von Resonanz (z.B.: «im Thema sein»; «einen Draht zur Gruppe haben») sowie erlebter Momente von Entfremdung (z.B.: «Situation, in der der Faden verloren geht»; «man von der Gruppe nicht gehört wird») bzw. dessen dialektisches Verhältnis beleuchtet werden.

Ziel der Untersuchung stellt die Identifikation von Resonanz- und Entfremdungspotenzialen aus Sicht von Weiterbildungsteilnehmenden dar. Ziel der Analyse für die Erwachsenenbildungsforschung und -praxis sind u.a. folgende Aspekte:

- Reflexion bzw. Bewusstwerdung von Resonanzerfahrungen vor dem Hintergrund einer zunehmenden Beschleunigung und Digitalisierung (in digitalen und analogen Umwelten),

- Reflexion bzw. Bewusstwerdung von Widersprüchen und Entfremdung, Identifikation von kollektiven Erfahrungsräumen/-momenten.

Das entwickelte heuristische Modell der RRS dient der Beschreibung von erlebter Resonanz und Entfremdung im Lehr-Lern-Geschehen. Hierbei geht es um die Herausbildung und den (bewussten) Einsatz von förderlichen Strategien zur Erhöhung der Ermöglichung von Resonanz. Die Handlungsweisen, die aus der Befragung der Weiterbildungsteilnehmenden im Umgang mit Beschleunigung und Digitalisierung hervorgingen, werden im Folgenden exemplarisch entfaltet.

Folgende zentrale Aspekte wurden im Spannungsfeld der Beschleunigung und Digitalisierung von den Befragten geäussert. Die Verortung, die im Material identifizierte Wahrnehmung, das Erleben und der Umgang mit Beschleunigungs- und Digitalisierungsphänomenen erstrecken sich kontextbezogen auf einem Kontinuum: 
- Reichweite: Zum einen wurden eine wahrgenommene Reichweitenvergrösserung, lebensbereichsübergreifende Entgrenzung und Flexibilisierung benannt. Zum anderen wurden unterschiedliche Formen, Ausprägungen und Ausmasse räumlicher und zeitlicher Begrenzungen, Stagnationen bzw. vorherrschenden Standardisierung sowie Ein- und Begrenzungen erfahren.

- Einfluss: Die wahrgenommene Einflussnahme auf das Geschehen, Verhaltens- und Handlungsmöglichkeiten erstrecken sich in sehr differenzierten Ausprägungen von einem hohen Mass an Selbstwirksamkeit(serwartung) bis hin zu geäusserten Befürchtungen des Kontrollverlusts bezüglich des eigenen Verhaltens, Reagierens und Agierens.

- Sinn: Die aus dem Material gesichteten Äusserungen hinsichtlich des Empfindens und des subjektiven Erlebens erweisen sich auf der Verhältnis- und Verhaltensebene in der Ausdeutung des jeweiligen Sinnbezugs als äusserst divergent: Diese Wahrnehmungen reichen von einer starken, sinnstiftenden Verinnerlichung von Normen, Werten und Zielen etc. bis hin zu einer (als fremdbestimmt empfundenen) Auferlegung; häufig verbunden mit dem Empfinden von Inkongruenz.

- (Selbst-)Zweck: Es wurden in der Befragung Aspekte deutlich, inwieweit eine (Selbst-)Zweckgebundenheit wahrgenommen wird: Deutungen und Handlungsformen richten sich hierbei sowohl auf die Aufrechterhaltung bzw. Erlangung von Individualität und (individuelle und kollektive) Aushandlungs- und Abgrenzungsprozesse als auch auf die Ausrichtung auf den sogenannten («ökonomisierten») Mainstream.

Die aus dem Interviewmaterial generierte temporale Resonanzstrategie 〈Vorlaufen〉 beispielsweise lässt sich durch folgende Handlungsweisen im Umgang mit Beschleunigung und Digitalisierung, die von den Befragten beschrieben wurden, skizzieren: Charakteristisch für diese Strategie des ,Vorlaufens' ist die kontinuierliche Wahrnehmung einer nicht zu stoppenden Beschleunigung, welcher es durch Bündelung von eigenen Ressourcen und deren gezielten, effizienzgetriebenen Einsatz zu begegnen gilt. Häufig finden sich diese Wahrnehmung und empfundener anspornender Druck in Handlungen der Überanpassung wieder: dem Antrieb schneller als die 
Beschleunigung zu sein - im Sinne einer Wettbewerbslogik und eines ökonomisierten Verständnisses und einer Output-Orientierung.

$\mathrm{Zu}$ der Resonanzstrategie〈Mitlaufen〉 lassen sich ebenso spezifische Handlungsweisen im Umgang mit Beschleunigung und Digitalisierung aus dem Datenmaterial identifizieren: Prägnant erscheinen Handlungsweisen der Befragten, welche (primär) intendieren, sich im Bereich des Mainstreams verorten zu lassen beziehungsweise die Interviewten veranlassen, sich mitreissen zu lassen und mit dem Strom zu schwimmen.

Die Resonanzstrategie 〈Innehalten〉 ist hingegen dadurch gekennzeichnet, dass sich das Verhalten und die Performanz an einer grossen Achtsamkeit ausrichten. Die Handlungsweisen sind überwiegend gegenwartsbezogen beziehungsweise konzentrieren sich auf eine Sache zurzeit. Handlungsweisen dienen zur Konzentration und Fokussierung und werden vom Multitasking abgegrenzt. Die letzte kategoriale Einordung stellt die Resonanzstrategie 〈Gegenlaufen〉 dar: Jene Handlungsweisen im Umgang mit Beschleunigung und Digitalisierung verdeutlichen beispielsweise eine bewusste Abhebung vom Mainstream im Sinne der Differenzierung und Distanzierung vom Kollektiv, dem man sich zugehörig fühlt (Eigengruppe), aber auch in Abgrenzung zu anderen Gruppierungen (《othering»). 


\begin{tabular}{|c|c|c|c|}
\hline $\begin{array}{l}\text { Temporale \& technoi- } \\
\text { de Strategien: } \\
\text { VORLAUFEN }\end{array}$ & $\begin{array}{l}\text { Temporale \& technoi- } \\
\text { de Strategien: } \\
\text { MITLAUFEN }\end{array}$ & $\begin{array}{l}\text { Temporale \& technoi- } \\
\text { de Strategien: } \\
\text { INNEHALTEN }\end{array}$ & $\begin{array}{l}\text { Temporale \& technoi- } \\
\text { de Strategien: } \\
\text { GEGENLAUFEN }\end{array}$ \\
\hline $\begin{array}{l}\text { Handlungsweisen: } \\
\text { - Überanpassung: } \\
\text { schneller als die Be- } \\
\text { schleunigung (Road } \\
\text { Runner) } \\
\text { - Antizipation: Vor- } \\
\text { ausgreifendes Han- } \\
\text { deln zur Bewahrung } \\
\text { von Kontrolle } \\
\text { - Effizienzsteigerung } \\
\text { - Ökonomisierung/ } \\
\text { Wettbewerbslogik: } \\
\text { Stillstand und v.a. } \\
\text { Rückschritt schei- } \\
\text { nen unerträglich } \\
\text { - Zeitliche und räum- } \\
\text { liche Reichweiten- } \\
\text { vergrösserung: } \\
\text { • Radikale Flexibili- } \\
\text { sierung } \\
\text { - Ständiger Ausbau } \\
\text { von Mobilität } \\
\text { - Pausen- und Lü- } \\
\text { ckenlernen }\end{array}$ & $\begin{array}{l}\text { Handlungsweisen: } \\
\text { - Mit dem Strom } \\
\text { schwimmen (Main- } \\
\text { stream) } \\
\text { - Divergente Anpas- } \\
\text { sung: sich oppor- } \\
\text { tunistisch wie die } \\
\text { Mehrheit verhalten } \\
\text { beim gleichzeitigen } \\
\text { Versuch, sich von } \\
\text { der Masse abzuhe- } \\
\text { ben } \\
\text { - Handlungsweisen } \\
\text { zur Begegnung } \\
\text { einer scheinbar } \\
\text { nicht abwendbaren } \\
\text { Getriebenheit } \\
\text { - Laissez-faire-Ein- } \\
\text { stellung: mit einer } \\
\text { Gelassenheit Ver- } \\
\text { änderung auf sich } \\
\text { zukommen lassen } \\
\text { - Weltzugewandte } \\
\text { Selbstverwirkli- } \\
\text { chung } \\
\text { - Stark erlebte } \\
\text { Fremdbestimmung; } \\
\text { Anwendung reak- } \\
\text { tiver Handlungs- } \\
\text { weisen }\end{array}$ & $\begin{array}{l}\text { Handungsweisen: } \\
\text { - Achtsamkeit: ge- } \\
\text { genwartsbezogene } \\
\text { Konzentration auf } \\
\text { eine Sache } \\
\text { - Regeneration: Still- } \\
\text { stand wird als wert- } \\
\text { voll empfunden } \\
\text { - Systematische Zeit- } \\
\text { planung } \\
\text { - Versuch einer Ab- } \\
\text { grenzung zwischen } \\
\text { Arbeit und Freizeit } \\
\text { (Work-Life-Balance) } \\
\text { - Bewusste Planung } \\
\text { und Nutzung von } \\
\text { (Aus-)Zeiten } \\
\text { - Situative Aufmerk- } \\
\text { samkeitssteue- } \\
\text { rung des Wahr- } \\
\text { nehmungs- und } \\
\text { Bewusstseinszu- } \\
\text { stands: } \\
\text { - Handlungsweisen } \\
\text { zur Aufrechterhal- } \\
\text { tung der Wach- } \\
\text { samkeit } \\
\text { - Aufmerksamkeit } \\
\text { für das eigene Er- } \\
\text { leben von Innen- } \\
\text { und Aussenwelt }\end{array}$ & $\begin{array}{l}\text { Handlungsweisen: } \\
\text { - Abhebung vom } \\
\text { Mainstream (Au- } \\
\text { thentizität) } \\
\text { - Abgrenzung und Dis- } \\
\text { tanzierung (Agieren) } \\
\text { - Bewahrung des } \\
\text { Status Quo: proakti- } \\
\text { ve Vorgehensweisen } \\
\text { zur Aufrechterhal- } \\
\text { tung von Konstanz } \\
\text { - Systematische Ein- } \\
\text { haltung von verein- } \\
\text { barten Normen und } \\
\text { Auszeiten: bei Nicht- } \\
\text { einhaltung folgt } \\
\text { eine Verweigerung/ } \\
\text { Zurückweisung } \\
\text { - Strikte Trennung } \\
\text { von Arbeit und } \\
\text { Freizeit } \\
\text { - Handlungsweisen } \\
\text { zur Entschleunigung } \\
\text { - Weltabgewandte } \\
\text { Selbstverwirkli- } \\
\text { chung } \\
\text { - Bewahrung von } \\
\text { Analogie/Vermei- } \\
\text { dung von Technolo- } \\
\text { gisierung } \\
\text { - Bedeutsamkeit } \\
\text { menschlicher Be- } \\
\text { ziehungen/direkter } \\
\text { persönlicher Kon- } \\
\text { takt }\end{array}$ \\
\hline
\end{tabular}

Tab. 4.: Relationale Resonanzstrategien im Umgang mit Beschleunigung und Digitalisierung (Wienberg 2019b; Wienberg i.V.). 
Bei der Betrachtung der unterschiedlichen Handlungsweisen, mit denen Beschleunigungs- sowie Technikphänomen begegnet wird und der Betrachtung, welche Strategien im Umgang damit angewandt werden, wird eine grosse Bandbreite deutlich. Zudem zeigt sich das dialektische Verhältnis von erlebter Resonanz und Entfremdung. Resonanz- und Entfremdungsmomente können demzufolge als eine Art «Scharnier» innerhalb der Bildungsbiografie begriffen werden. Erlebte Momente von Resonanz und Entfremdung nehmen somit eine weichenstellende Rolle für das Lernen über den Lebenslauf - und somit eine zentrale Rolle für die Erwachsenenbildung/Weiterbildung - ein.

\section{Resümee \& Ausblick}

Die zuvor erläuterten Beschleunigungs- und Digitalisierungstendenzen können für einige Menschen im Zuge der zunehmenden Steigerungslogik einer Anverwandlung von (subjektiven) Resonanzerleben entgegentreten - zumal ein grösserer Teil der erwachsenen Bevölkerung über wenig ausgeprägte digitale Praktiken verfügt und sich als wenig kompetent im Umgang mit digitalen Herausforderungen betrachtet.

Veränderte Anforderungen an das Subjekt treten aufgrund des digitalen Wandelns auf, beispielsweise in der Gestalt von flexibleren Orts- und Zeitnutzung auch im Hinblick auf unterschiedliche Bildungsaktivitäten und digitalen Alltagspraktiken etwa in den Bereichen Mobilität, Arbeitswelt, Kommunikation oder Finanzen (Buddeberg und Grotlüschen 2020).

Diese Anforderungen stellen verschiedene Bevölkerungsgruppen in unterschiedlichem Masse vor Herausforderungen. Es sind aber keinesfalls nur als bildungsfern (Brüning und Kuwan 2002) oder als bildungsarm (Baumert et al. 2018) bezeichnete Gruppen, die von seltener Praxis und geringen Kompetenzen berichten. Die Gefahr von Teilhabeausschlüssen und daraus resultierend konkrete Bildungsbedarfe betreffen auch Erwachsene mit höherer formaler Bildung.

Hierbei kann das Erleben von Resonanz- und Entfremdungsmomenten im Umgang mit dem Beschleunigungsphänomen als «Scharnier» innerhalb der Bildungsbiografie und insbesondere bei Transitionen angesehen werden. 
Die Heterogenität von Lernenden und deren sehr unterschiedlichen Vorwissen, Bedarfen sowie Motivlagen ist kennzeichnend für den Bereich der Erwachsenenbildung. Ein breites Spektrum der mannigfaltigen Handlungsweisen fand sich auch in den jeweiligen Resonanzstrategien im Umgang mit Beschleunigung und Digitalisierung bei den Befragten wieder. Sowohl im Hinblick auf (digitale) Bildungsaktivitäten als auch auf lebensalltägliche digitale Praktiken zeigen sich im Erleben und im Umgang grosse Unterschiede. Das heuristische Modell der Relationalen Resonanzstrategien zur Beschreibung von Momenten erlebter Resonanz und Entfremdung sowie resonanzermöglichenden Strategien verdeutlichen das Spektrum an Handlungsweisen im Umgang mit Beschleunigung und Digitalisierung.

Einen Beitrag den die Resonanztheorie für die Erwachsenenbildung/ Weiterbildung leisten kann, ist eine spezifische Sicht auf die Wahrnehmung, das Erleben und den Umgang mit Beschleunigung und Digitalisierung in Form von resonanzstabilisierenden oder -ermöglichenden Strategien. Eine resonanztheoretische Ausdeutung vermag hierbei eine Betrachtung spezifischer Antwortbeziehungen (Subjekt-Welt-Beziehungen) im subjektiven Erleben und in der Wahrnehmung zu ermöglichen. Der Fokus der resonanztheoretischen Betrachtung liegt auf der Art und Weise, wie die Befragten in Beziehung zum Kurs(-inhalt), zu den Teilnehmenden, zu den Kursleitenden etc. - kurzum zu ihrer (Lern-)Umwelt treten.

Im Zuge der Untersuchung wurde als Forschungsdesiderat deutlich, dass erlebte Resonanz vor dem Hintergrund von zunehmender Beschleunigung und Digitalisierung in der Erwachsenenbildung/Weiterbildung aus resonanztheoretischer Sicht relativ unbeforscht ist - theoretische und empirische Untersuchungen bestehen bislang ausschliesslich im schulischen Kontext (z.B. unter dem Begriff der «Resonanzpädagogik»).

Das entwickelte heuristische Modell der RRS dient der Beschreibung von erlebter Resonanz und Entfremdung im Lehr-Lern-Geschehen. Hierbei geht es um die Herausbildung und den (bewussten) Einsatz von förderlichen Strategien zur Erhöhung der Ermöglichung von Resonanz. Der resonanztheoretische Ansatz soll auf der theoretischen Ebene für die Erwachsenenbildung/Weiterbildung fruchtbar und anschlussfähig an aktuelle Diskurse gemacht werden. 


\section{Literatur}

Ahrens, Daniela, und Michael Gessler. 2018. «Von der Humanisierung zur Digitalisierung: Entwicklungsetappen betrieblicher Kompetenzentwicklung». In Kompetenzentwicklung in analogen und digitalisierten Arbeitswelten, hrsg. v. Daniela Ahrens, und Gabriele Molzberger. Berlin: Springer.

Aufenanger, Stefan. 2018. «Lehren und Lernen mit digitalen Medien - Ein kurzer Forschungsüberblick». In Die digitale $R^{*}$ Evolution? Herausforderungen für Berufliche Bildung und Weiterbildung. Dokumentation der Bundestagung Berufliche Bildung und Weiterbildung, 23. und 24. November 2017, 50-52. https://www. gew.de/index.php?eID=dumpFile\&t $=f \& f=67840 \&$ token=9a6f27fllac3f3ab3f 4 e8af855b8821c187184b5\&sdownload=\&n=201804_Tagungsdoku_Digitalisierung_BBWB-web.pdf.

Arbeitsstab Forum Bildung (Hrsg.). 2001. Lernen - ein Leben lang. Vorläufige Empfehlungen und Expertenbericht. Bonn. https://www.pedocs.de/volltexte/2008/188/pdf/bandog.pdf.

Baer, Udo. 2005. «Resonanz». In Bausteine einer kreativen Sozio- und Psychotherapie. Ausgewählte Beiträge 1991-2005, hrsg. v. Udo Baer, und Gabriele Frick-Baer, 36-64. Neunkirchen-Vluyn: Affenkönigverlag.

Baumert, Jürgen, Kai Maaz, Josefine Lühe, und Stefan Schulz. 2018. «Bildungsungleichheit und Bildungsarmut - Der Beitrag von Large-Scale-Assessments». In Handbuch Bildungsarmut, hrsg. V. Gudrun Quenzel und Klaus Hurrelmann, 261-85. Wiesbaden: Springer VS.

Beck, Ulrich. 1986. Risikogesellschaft - auf dem Weg in eine andere Moderne. Frankfurt a. M.: Suhrkamp.

Beljan, Jens. 2017. Schule als Resonanzraum und Entfremdungszone. Eine neue Perspektive auf Bildung. Weinheim: Beltz.

Benjamin, Walter. 1991. «Über einige Motive bei Baudelaire». In Gesammelte Schriften, Band I.2, hrsg. v. Rolf Tiedemann, Hermann Schweppenhauser, 60553. Frankfurt a. M.: Suhrkamp.

Blossfeld, Hans-Peter et al. 2017. Bildung 2030 - veränderte Welt. Fragen an die Bildungspolitik. Gutachten, hrsg. v. Vereinigung der Bayerischen Wirtschaft e.V. Münster: Waxmann.

BMBF und Kultusministerkonferenz. 2016. Grundsatzpapier zur Nationalen Dekade für Alphabetisierung und Grundbildung 2016-2026. Den funktionalen Analphabetismus in Deutschland verringern und das Grundbildungsniveau erhöhen. https://www.alphadekade.de/files/O1_Grundsatzpapier zur Nationalen Dekade Alphabetisierung und Grundbildung_final.pdf.

Brüning, Gerhild, und Helmut Kuwan. 2002. Benachteiligte und Bildungsferne Empfehlungen für die Weiterbildung. Bielefeld: wbv.

Buddeberg, Klaus, und Anke Grotlüschen. 2020. «Literalität, digitale Praktiken und Grundkompetenzen». In LEO 2018. Leben mit geringer Literalität, hrsg. v. Anke Grotlüschen und Klaus Buddeberg. Bielefeld: wbv Media, 197-225. 
Burchert, Joanna, und Rasmus Grobe. 2017. «Herausforderungen bei der Implementierung digital gestützter beruflicher Weiterbildung. Die Sicht von WeiterbildnerInnen und BildungsmanagerInnen auf Strukturen, kulturelle Praktiken und Agency». In Magazin erwachsenenbildung.at. http://www.erwachsenenbildung.at/magazin/17-30/meb17-30.pdf.

Eickelmann, Birgit, Wilfried Bos, Julia Gerick, Frank Goldhammer, Heike Schaumburg, und Knut Schwippert et al., Hrsg. 2019. ICILS 2018 \#Deutschland. Computer- und informationsbezogene Kompetenzen von Schülerinnen und Schülern im zweiten internationalen Vergleich und Kompetenzen im Bereich Computational Thinking. Münster: Waxmann.

Fraillon, Julian, John Ainley, Wolfram Schulz, Tim Friedman, und Daniel Duckworth. 2019. Preparing for life in a digital world. IEA International Computer and Information Literacy Study 2018 International Report. International Association for the Evaluation of Educational Achievement (IEA). Amsterdam. https:// www.iea.nl/sites/default/files/2019-11/ICILS\%202019\%20Digital\%20final\%20 04112019.pdf.

Gärtner, Christian, und Günther Ortmann. 2017. «Organisation und Institution». In Handbuch Körpersoziologie, Band 2: Forschungsfelder und Methodische Zugänge, hrsg. v. Robert Gugutzer, Gabriele Klein, Michael Meuser, 175-86. Wiesbaden: Springer.

Grotlüschen, Anke, und Klaus Buddeberg, Hrsg. 2020. LEO 2018. Leben mit geringer Literalität. Bielefeld: wbv Media

Glaser, Barney Gailand. 1978. Theoretical Sensitivity: Advances in the Methodology of Grounded Theory. Mill Valley (Ca.): Sociology Press.

Haberzeth, Erik, und Irena Sgier. 2019. Digitalisierung und Lernen: Gestaltungsperspektiven für das professionelle Handeln in der Erwachsenenbildung und Weiterbildung [Forum Hochschuldidaktik und Erwachsenenbildung, Bd. 8]. Bern: hep verlag ag.

Hirsch-Kreinsen, Hartmut. 2016. «Zum Verhältnis von Arbeit und Technik bei der Industrie 4.0». APuZ 18-19/2016, 10-16.

Horkheimer, Max, und Theodor W. Adorno. 1969. Dialektik der Aufklärung: Philosophische Fragmente. Frankfurt a. M.: Fischer Taschenbücher.

Hösel, Fanny, und Carlo Terzaroli. 2017. «Work Transitions in Adulthood: An Analytical Tool for Comparative Studies». In Adult learning and education in international contexts. Future challenges for its professionalization: comparative perspectives from the 2016 Wurzburg Winter School, hrsg. v. Regina Egetenmeyer, Sabine Schmidt-Lauff, und Vanna Boffo, 131-46. Frankfurt am Main: Peter Lang (Studies in Pedagogy, Andragogy, and Gerontagogy, Vol. 69).

Institut für Arbeitsmarkt- und Berufsforschung (IAB). 2019. IAB-Stellungnahme. Digitalisierung: Herausforderungen für die Aus- und Weiterbildung in Deutschland. Nürnberg: Institut für Arbeitsmarkt- und Berufsforschung der Bundesagentur für Arbeit. 
Jaeggi, Rahel. 2005. Entfremdung. Zur Aktualität eines sozialphilosophischen Problems. Frankfurt a. M.: Campus.

Jaeggi, Rahel. 2014. Kritik der Lebensformen. Berlin: Suhrkamp.

Kammer, Matthias. 2018. DIVSI U25-Studie - Euphorie war gestern: Die «Generation Internet» zwischen Glück und Abhängigkeit. DIVSI Deutsches Institut für Vertrauen und Sicherheit im Internet. https://www.divsi.de/publikationen/studien/ divsi-u25-studie-euphorie-war-gestern/.

Käpplinger, Bernd. 2016. «Weiterbildung 3.1 kommt vor Weiterbildung 4.0». Personalführung 12/2016-1/2017, 20-26.

Kessl, Fabian. 2017. «Individuum und Gesellschaft». In Soziale Arbeit - Kernthemen und Problemfelder hrsg. v. Fabian Kessl, Elke Kruse, Sabine Stövesand, und Werner Thole, 52-60. Oplden, Toronto: Barbara Budrich Verlag.

King, Vera, und Benigna Gerisch, Hrsg. 2009. Zeitgewinn und Selbstverlust. Folgen und Grenzen der Beschleunigung. Frankfurt am Main: Campus Verlag.

Klieme, Eckhard, und Detlev Leutner. 2006. Kompetenzmodelle zur Erfassung individueller Lernergebnisse und zur Bilanzierung von Bildungsprozessen. Überarbeitete Fassung des Antrags an die DFG auf Einrichtung eines Schwerpunktprogramms.

Klieme, Eckhard, und Johannes Hartig. 2008. «Kompetenzkonzepte in den Sozialwissenschaften und im erziehungswissenschaftlichen Diskurs». In Kompetenzdiagnostik. Zeitschrift für Erziehungswissenschaft, hrsg. v. Manfred Prenzel, Ingrid Gogolin, und Heinz-Hermann Krüger, 11-32. Wiesbaden: VS Verlag für Sozialwissenschaften GWV Fachverlage GmbH.

Korunka, Christian, und Bettina Kubicek. 2013. «Beschleunigung im Arbeitsleben - neue Anforderungen und deren Folgen». In Immer schneller, immer mehr: Psychische Belastung bei Wissens- und Dienstleistungsarbeit, hrsg. v. Bundesanstalt für Arbeitsschutz und Arbeitsmedizin Gisa Junghanns, und Martina Morschhäuser, 17-40. Berlin: Springer.

Langemeyer, Ines. 2015. Das Wissen der Achtsamkeit. Kooperative Kompetenz in komplexen Arbeitsprozessen. Münster: Waxmann.

Lengersdorf, Diana. 2017. «Arbeit». In Handbuch Körpersoziologie, Bd. 2, hrsg. v. Robert Gugutzer, Gabriele Klein, und Michael Meuser 17-28. Wiesbaden: Springer.

Lennon, Mary Louise, und Claudia Tamassia. 2016. "The Development of the PIAAC Cognitive Instruments》. In Technical Report of the Survey of Adult Skills (PIAAC), hrsg. v. OECD. 2nd Edition, Chapter 2.

Marcuse, Herbert. 1998. Der eindimensionale Mensch. Studien zur Ideologie der fortgeschrittenen Industriegesellschaft. München: Deutscher Taschenbuch Verlag.

Marx, Karl. 1996. «Die entfremdete Arbeit». In Konflikttheorien. Friedens- und Konfliktforschung. Vol.2/1996. Wiesbaden: Springer Fachmedien, 155-166.

Meyer-Drawe, Käte. 2008. Diskurse des Lernens. München: Wilhelm Fink Verlag. 
Negt, Oskar. 1990. «Überlegungen zur Kategorie ,Zusammenhang' als einer gesellschaftlichen Schlüsselqualifikation». REPORT Literatur- und Forschungsreport Weiterbildung 26 (4), 11-19.

Negt, Oskar. 1993. «Wir brauchen eine zweite, gesamtdeutsche Bildungsreform». Gewerkschaftliche Monatshefte 44 (11), 657-68.

Nygren, Thomas, und Mona Guath. 2019. "Swedish teenagers' difficulties and abilities to determine digital news credibility». Nordicom Review 40 (1), $23-42$.

Paech, Niko. 2012. Befreiung vom Überfluss. Auf dem Weg in die Postwachstumsökonomie. München: Oekom Verlag.

Pietraß, Manuela. 2013. "Medienwandel und Generationenunterschiede. Zur Haltbarkeit der These von den „Digital Natives"». In Familienleben: Entgrenzt und vernetzt? hrsg. v. Ulrike Wagner. Neue Ausg. München: kopaed (Interdisziplinäre Dikurse, 7), 77-86.

Reder, Stephen (2017): Adults' Engagement in Reading, Writing and Numeracy Practices. Portland State University. Portland (Applied Linguistics Faculty Publications and Presentations, 22). http://pdxscholar.library.pdx.edu/ling_fac/22.

Renn, Klaus. 2009. «Resonanz». In Wörterbuch der Psychotherapie. 2. Auflage, hrsg. v. Gerhard Stumm, und Alfred Pritz, 593-94. Wien, New York: Springer.

Ribolits, Erich. 2007. «Führe mich sanft. Beratung, Coaching \& Co. - die postmodernen Instrumente der Gouvernementalität». Schulheft (32) 126.

Riekmann, Wibke, und Anke Grotlüschen. 2011. «Das Gemeinsame und das Trennende der Kompetenzbegriffe». In Die Bildung der Erwachsenen. Perspektiven und Utopien; für Peter Faulstich zum 65. Geburtstag. Unter Mitarbeit von Peter Faulstich, hrsg. v. Svenja Möller, Christine Zeuner und Anke Grotlüschen. Weinheim, München: Juventa-Verlag (Zukünfte), 62-71.

Ritzer, Georg. 2017. «Die McDonaldisierung, Amerikanisierung und Globalisierung. Eine vergleichende Analyse». In Globales Amerika? Die kulturellen Folgen der Globalisierung, hrsg. v. Ulrich Beck et al., 44-68. Bielefeld: transcript Verlag.

Rosa, Hartmut. 2005. Beschleunigung. Die Veränderung der Zeitstrukturen in der Moderne. Frankfurt a. M.: Suhrkamp.

Rosa, Hartmut. 2009. «Kritik der Zeitverhältnisse. Beschleunigung und Entfremdung als Schlüsselbegriffe der Sozialkritik». In Was ist Kritik?, hrsg. v. Rahel Jaeggi, und Tilo Wesche, 23-54. Frankfurt a. M.: Suhrkamp.

Rosa, Hartmut. 2011. "Entfremdung in der Spätmoderne. Umrisse einer Kritischen Theorie der sozialen Beschleunigung». In Nachrichten aus den Innenwelten des Kapitalismus, hrsg. v. Cornelia Koppetsch, 221-52. Wiesbaden: Springer.

Rosa, Hartmut. 2012. "Arbeit und Entfremdung》. In Kapitalismustheorie und Arbeit. Neue Ansätze soziologischer Kritik, hrsg. v. Klaus Dörre, 410-20. Frankfurt a. M.: Campus Verlag. 
Rosa, Hartmut. 2014. Beschleunigung. Die Veränderung der Zeitstrukturen in der Moderne. 10. Auflage. Frankfurt a. M.: Suhrkamp.

Rosa, Hartmut. 2016a. Weltbeziehungen im Zeitalter der Beschleunigung. Umrisse einer neuen kritischen Gesellschaftskritik. Berlin: Suhrkamp.

Rosa, Hartmut. 2016b. Resonanz. Eine Soziologie der Weltbeziehung. Berlin: Suhrkamp.

Schmid, Ulrich, Lutz Goetz, und Julia Behrens. 2017. Monitor Digitale Bildung. Die Weiterbildung im digitalen Zeitalter. Gütersloh: Bertelsmann Stiftung.

Schmidt-Lauff, Sabine. 2004. «Lernzeitstrategien - betriebliche Realitäten und individuelle Wünsche». Report (27), 1/ 2004, 124-31.

Schmidt-Lauff, Sabine. 2012. "Grundüberlegungen zu Zeit und Bildung». In Zeit und Bildung. Annäherungen an eine zeittheoretische Grundlegung, hrsg. v. Sabine Schmidt-Lauff, 11-60. Münster: Waxmann.

Schulmeister, Rolf. 2012. «Vom Mythos der Digital Natives und der Net Generation». Berufsbildung in Wissenschaft und Praxis (3), 42-46.

Sennet, Richard. 1998. Der flexible Mensch. Die Kultur des neuen Kapitalismus. Berlin: Berlin-Verlag.

Simmel, Georg. 1919. «Der Begriff und die Tragödie der Kultur». In Philosophische Kultur. Leipzig. Alfred Kröner Verlag, 223-253.

Strauss, Anselm, und Juliet Corbin. 1996. Grundlagen qualitativer Sozialforschung. Weinheim: Beltz.

Weber, Max. 2016. Die protestantische Ethik und der «Geist» des Kapitalismus. Neuausgabe der ersten Fassung von 1904-05. Wiesbaden: Springer Verlag.

Wicht, Alexandra, Clemens Lechner, und Beatrice Rammstedt. 2018. «Wie steht es um die Digitalkompetenz deutscher Erwachsener? Eine empirische Analyse mit dem Programme for the International Assessment of Adult Competencies (PIAAC)». In: Mensch und Gesellschaft im digitalen Wandel. Psychologie, Gesellschaft, Politik 2018, hrsg. v. Berufsverband Deutscher Psychologinnen und Psychologen. 1. Auflage. Berlin: Deutscher Psychologen Verlag, 15-25.

Wienberg, Jana. 2018. «Eine resonanztheoretische Betrachtung von Beratung». In Beratung im Kontext des Lebenslangen Lernens. Konzepte, Organisation, Politik, Spannungsfelder, hrsg. v. Olaf Dörner, Carola Iller, Ingeborg Schüßler, Cornelia Maier-Gutheil, und Christiane Schiersmann, 35-46. Opladen, Berlin, Toronto: Barbara Budrich Verlag.

Wienberg, Jana. 2019a. "Resonanz in der Arbeitswelt - keine Frage des Alters». In Das Politische in der Erwachsenenbildung, hrsg. v. Anke Grotlüschen, Sabine Schmidt-Lauff, Silke Schreiber-Barsch, und Christine Zeuner, 190-94. Frankfurt a. M.: Wochenschau Verlag. 
Wienberg, Jana (2019b). «Erlebte Resonanz im Weiterbildungsgeschehen - eine empirische Annäherung». In Erwachsenenbildung und Lernen in Zeiten von Globalisierung, Transformation und Entgrenzung, hrsg. v. Olaf Dörner, Carola Iller, Ingeborg Schüßler, Heide von Felden, und Sebastian Lerch. Opladen, Berlin, Toronto: Barbara Budrich Verlag.

Wienberg, Jana (i.V.): ,Catch me if I can' - Erlebte Momente von Resonanz im Weiterbildungsgeschehen und die Herausbildung von Relationalen Resonanzstrategien (RRS). Eine qualitative Untersuchung im Feld der betrieblichen Weiterbildung und der konfessionellen Erwachsenenbildung. Habilitationsschrift.

Wolf, Karsten D., und Ilka Koppel. 2017. «Digitale Grundbildung: Ziel oder Methode einer chancengleichen Teilhabe in einer mediatisierten Gesellschaft? Wo wir stehen und wo wir hin müssen». erwachsenenbildung.at (30), 1-11.

Zabal, Anouk, Silke Martin, Anja Klaukien, Beatrice Rammstedt, Jürgen Baumert, und Eckhard Klieme. 2013. "Grundlegende Kompetenzen der erwachsenen Bevölkerung in Deutschland im internationalen Vergleich». In: Grundlegende Kompetenzen Erwachsener im internationalen Vergleich. Ergebnisse von PIAAC 2012, hrsg. v. Beatrice Rammstedt, 31-76. Münster: Waxmann.

Zeuner, Christine. 2009. «Zur Bedeutung gesellschaftlicher Kompetenzen im Sinne eines kritischen bildungstheoretischen Ansatzes». In Eigen-Sinn und Widerstand. Kritische Beiträge zum Kompetenzentwicklungsdiskurs, hrsg. v. Axel Bolder, und Rolf Dobischat. 1. Auflage, 260-81. Wiesbaden: VS Verlag für Sozialwissenschaften GWV Fachverlage GmbH, Wiesbaden (Bildung und Arbeit, Bd. 1). 